\title{
Visualization and Quantification of Geometric Diversity in Metal-Organic Frameworks
}

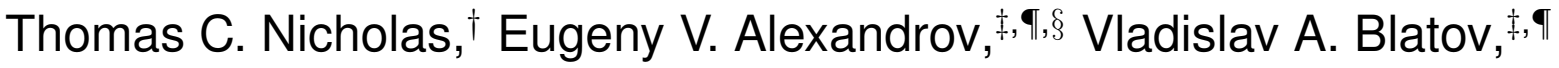 \\ Alexander P. Shevchenko, ${ }^{\ddagger}, \delta$ Davide M. Proserpio, ${ }^{*}, \|, \neq$ Andrew L. \\ Goodwin, ${ }^{*, \dagger}$ and Volker L. Deringer ${ }^{*, \dagger}$ \\ $\dagger$ Department of Chemistry, Inorganic Chemistry Laboratory, University of Oxford, Oxford \\ OX1 3QR, United Kingdom \\ $\ddagger$ Samara Center for Theoretical Material Science (SCTMS) Samara State Technical \\ University, Molodogvardeyskaya St. 244, Samara 443100, Russian Federation \\ ISamara University, Ac. Pavlov St. 1, 443011 Samara, Russian Federation \\ $\S$ Samara Branch of P.N. Lebedev Physical Institute of the Russian Academy of Science, \\ Novo-Sadovaya St. 221, Samara 443011, Russian Federation \\ ||Dipartimento di Chimica, Università degli Studi di Milano, 20133 Milano, Italy \\ E-mail: davide.proserpio@unimi.it; andrew.goodwin@chem.ox.ac.uk; \\ volker.deringer@chem.ox.ac.uk
}

\begin{abstract}
With ever-growing numbers of metal-organic framework (MOF) materials being reported, new computational approaches are required for a quantitative understanding of structureproperty correlations in MOFs. Here we show how structural coarse-graining and embedding ("unsupervised learning") schemes can together give new insight into the geometric diversity of MOF structures. Based on a curated dataset of 1,262 reported experimental structures, we automatically generate coarse-grained and rescaled representations which we couple to a kernel-based similarity metric and to widely used embedding schemes. This approach allows us to visualize the breadth of geometric diversity within individual topologies, and to quantify the distributions of local and global similarities across the structural space of MOFs. The methodology is implemented in an openly available Python package, and is expected to be useful in future high-throughput studies.
\end{abstract}

\section{Introduction}

A cornucopia of experimentally determined crystal structures is described in continually expanding databases. ${ }^{1,2}$ These databases are now reaching sufficient sizes to provide an opportunity for extracting structure-property relationships based on data mining and machine learning (ML), in principle. ${ }^{3-5}$ Establishing these relationships, however, is a non-trivial task because there are multiple ways by which crystal structures can be represented, compared, and analyzed. This challenge is particularly acute for metal-organic frameworks (MOFs) where the diversity of both the metal centers ("nodes") and the organic linkers gives rise to considerable structural complexity. ${ }^{6-9}$

One of the ways in which MOFs are commonly described is in terms of their topology; that is, the connectivity between nodes and linkers. Topological analysis routines are wellestablished and are implemented in automated computer packages, such as ToposPro ${ }^{10}$ and Systre ${ }^{11}$ and have been found to be useful predictors for a number of material properties. For 
example, by considering the deformability afforded by a given topology (i.e., the ability of a framework to distort geometrically without disrupting the net connectivity), one may predict the rigidness of a framework, ${ }^{12}$ the tendency to interpenetrate, ${ }^{13}$ and elastic properties. ${ }^{14,15}$ Ultimately, by exploiting knowledge of how a given choice of node and linker will give rise to a particular topology, one can hope to design new MOFs. ${ }^{16-18}$

Yet, two MOFs with the same topology may have very different network geometries. By the latter term, we mean the spatial arrangement of local atomic environments in a MOF, including variations in bond lengths, angles, and longer-range ordering - all of which may affect material properties. For example, positive and negative thermal expansion can be switched in MOFs of a given topology simply by varying their geometry. ${ }^{19}$ By their very nature, however, each of the established geometric descriptors will cover only individual aspects of the structure; two MOFs with similar metal-linker distances might have different porosities, for example.

Atom-density-based representations offer an alternative means of quantifying the geometric similarity of crystal structures. ${ }^{20-22}$ One class of such metrics originated in the field of $\mathrm{ML}$ for physics and chemistry applications, where the development of structural descriptors for atomistic structure is one of the central research tasks. ${ }^{23-27}$ In particular, the Smooth Overlap of Atomic Positions (SOAP) descriptor was developed initially in the context of fitting machinelearned interatomic potentials. ${ }^{28}$ Subsequently, it was shown how similarity kernels based on the SOAP formalism may be used to analyze the structural similarity for molecular and bulk periodic structures. ${ }^{29}$

By coupling to ML techniques such as dimensionality reduction and data clustering, one can begin to navigate complex configurational spaces, ${ }^{30-32}$ and search for underlying structure-property relationships. ${ }^{33-38}$ We have recently demonstrated that a combination of coarse-graining, re-scaling, and SOAP analysis enables geometric comparison between very different classes of materials, exemplified us- ing a database of $\mathrm{AB}_{2}$ hybrid and inorganic networks. ${ }^{39}$ Other studies have emphasized the usefulness of unsupervised ${ }^{31}$ and supervised ${ }^{5}$ ML for MOFs, and very recently local coordination environments were used as features for predicting oxidation states in these materials. ${ }^{40}$

Here, with a view to facilitate further quantitative studies of geometric structure and structure-property relationships in MOFs, we describe a generalized coarse-graining approach for such purposes and its Python implementation - expanding widely on initial work in ref 39. Using a curated test set of fourconnected, $\mathrm{AB}_{2}$ coordination networks, we validate structure outputs of our implementation using the well-established ToposPro topology analysis, and then discuss examples of structural and chemical analysis that are enabled by our approach.

\section{Methodology}

The computational methodology can be separated into two stages: structure processing and structure analysis (Figure 1). Each stage will be discussed in turn in the following subsections. References to functions contained within the code will be highlighted in typewriter typeface throughout.

\section{Structure Processing}

The main structure-processing routines of our implementation are handled by a Python class called Structure. It uses Pymatgen ${ }^{41}$ to parse and store information in the Crystallographic Information File (CIF) format. All subsequent structure-processing tasks are then callable as attributes of the Structure class, as follows.

getSites()

Initially, the atomic species are classified into two categories: A (metal) sites, and "other" sites. In the absence of user-specified categories, elements are sorted according to the IUPAC International Chemical Identifier classification: ${ }^{42}$ metals are assigned to $\mathrm{A}$ sites, nonmetals to "other". In the present work, we focus on the geometric structure of the underlying nets (as opposed to also considering the energetic stability provided by guest ions 


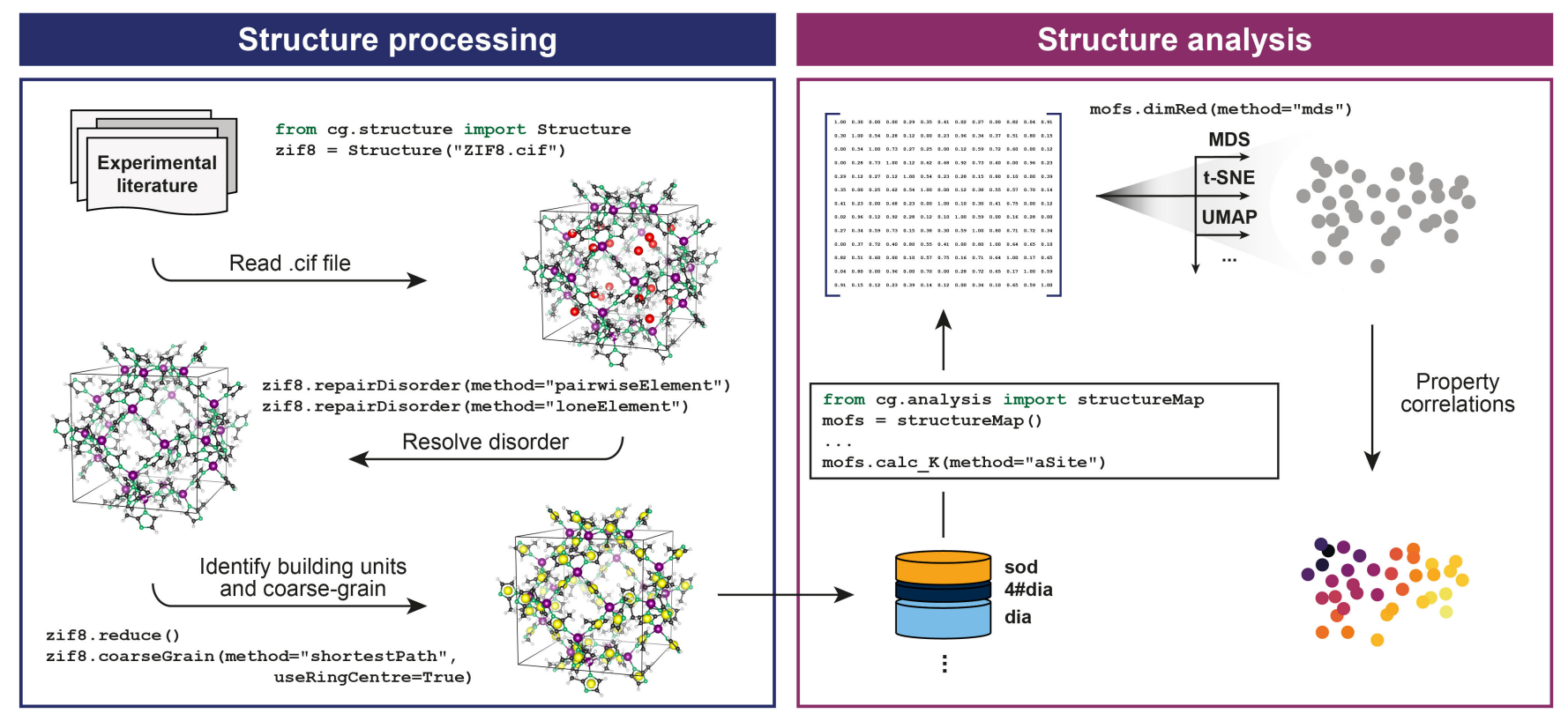

Figure 1 Methodology for processing and analyzing MOF structure datasets using a cg-SOAP-based approach (cf. ref 39). Left: Structures from the experimental literature may first need to be "cleaned-up" for analysis. Here, as in ref 39 , ZIF-8 is used as an example: the H-disorder is resolved using average positions of half-occupancy sites, and guest atoms in the pores are removed. The structure is then reduced to its constituent building blocks, viz. $12 \mathrm{Zn}^{2+}$ A sites (purple) and 24 methyl imidazolate B sites; the latter are coarse-grained using connectivity graphs (here, identifying the five-membered imidazolate rings as the B-site centers; yellow). Right: The SOAP kernel is used to determine the similarity between all coarse-grained and rescaled structures in the database, and the result is visualized using dimensionality reduction. The resultant 2-D structure map (sketched here in a purely schematic way) may then be interpreted by correlating the locations of data points with structural and physical properties. 
and molecules), and therefore we remove nonframework species. Accordingly, exceptions are made for the alkali and alkaline-earth metals (excluding $\mathrm{Li}$ and $\mathrm{Be}$ ) during the species sorting algorithm; they are often found as nonframework species, and are therefore removed from the structure. B, Si, and P are also sorted separately, because there are many instances in which they behave as either A or "other" sites (e.g. boron is an $A$ site in boron imidazolate frameworks, ${ }^{43}$ but a non-framework-site atom when a part of a $\mathrm{BF}_{4}^{-}$anion). Therefore, if the element is bound to $\mathrm{O}$ or $\mathrm{N}$ - indicative of Lewis-acidic-type behavior - it is assigned as an $\mathrm{A}$ site.

\section{repairDisorder()}

Site disorder occurs frequently in MOFs, and it is commonly modeled crystallographically using split sites with partial occupancies. $^{44}$ To enable structure-processing tasks that require discrete atomic positions, partially occupied (or disordered) sites are simplified using three algorithms, callable with the Structure.repairDisorder() method. First, atoms of the same species found within a given distance range (0-0.7 $\AA$ by default) are replaced by an atom of the same type, situated on the average of their positions. The remaining two routines deal with delocalized electron density associated with guest species within framework pores, which is often modeled by clusters of (fictitious) oxygen atoms. These clusters are removed by identifying either oxygen atoms that only have oxygen nearest neighbors, or oxygen atoms that have no neighbors at all within a set cut-off radius. Not all disorder needs to be resolved for the general coarse-graining procedure to work; the minimum requirement is that individual molecules (e.g. organic ligands) do not overlap. However, unresolved disorder may affect the placement of the discrete place-holder atoms (e.g. by skewing the centroid of a given molecule).

reduce ()

Each unique building block fragment, or ligand, is then identified by a "nearest-neighbor crawl" algorithm by calling the Structure.reduce() method. The default neighboring-sites routine used is CrystalNN, ${ }^{45}$ as implemented in Pymatgen. ${ }^{41}$ In short, CrystalNN uses Voronoi decomposition to assign weights to an initial list of neighbors within a hard cut-off radius, and then normalizes and assigns probabilities to each unique weighting according to a smooth cut-off function. With this definition, a building block fragment list is initialized with a given atom from a given building block type, and if another atom is both a nearest-neighbor and of the same building block type, it is added to the list. These neighbors are then searched for their respective nearest-neighbors, and the process is iterated (thereby "crawling" round the ligand) until the list size converges. Once converged, the atomic species, positions, and connectivity (in the form of a connectivity graph) of the fragment are stored as a buildingUnit class instance, and appended to the Structure.BUs class attribute. The algorithm repeats until all atoms have been classified.

coarseGrain()

To coarse-grain the building blocks, a discrete bonding center must first be defined. There is often not one unique choice for this. The simplest definition is to take the geometric centroid of all of the atomic positions in the building unit (i.e., not weighted by the atomic masses). This parallels methodology used in both crystal net determination (often referred to as "equilibrium" or "barycentric" placement), ${ }^{11}$ and coarse-grained molecular dynamic simulations. ${ }^{46}$ The buildingUnit class stores the connectivity of a given building unit as a graph object using the NetworkX package. ${ }^{47}$ This will enable the class to be extended to include alternative definitions of bonding centers, to which we will dedicate future work.

The building unit connectivity is defined as the number of nearest-neighbor atoms of a different site-type that are connected to it. All fragments with connectivity $>1$ (thereby distinguishing non-framework species, such as solvents, from the B sites) are coarse-grained by placing a dummy atom at the chosen bonding center, and removing all other atoms. Finally, all atoms of a given building-block type are as- 
signed to the same atomic species. The processed structures can then be output in CIF format.

\section{Structure Analysis}

Studying large, complex datasets requires generalized analysis routines. At the core of our analysis class, structureMap, structures are compared using $\mathrm{SOAP}^{28}$ which we apply to coarse-grained structural models (indicated by "cg-SOAP"). ${ }^{39}$ Using dimensionality reduction algorithms, configurations can be visualized and the relationships between them analyzed.

\section{cg-SOAP}

The SOAP kernel measures the similarity of pairs of atomic environments. ${ }^{28}$ Formally, for each atom, $\alpha$, an atomic density, $\rho_{\alpha}(\mathbf{r})$, is constructed with a sum of Gaussians of broadness $\sigma$, centered on each neighbor, $\beta$, of $\alpha$ (as well as on $\alpha$ itself):

$$
\rho_{\alpha}(\mathbf{r})=\sum_{\beta} \exp \left(-\frac{\left(\mathbf{r}_{\alpha}-\mathbf{r}_{\alpha \beta}\right)^{2}}{2 \sigma^{2}}\right) .
$$

The SOAP kernel is then defined as the overlap integral of any two neighbor densities, integrated over all three-dimensional rotations $\hat{R},{ }^{28}$

$$
k(\alpha, \beta)=\int \mathrm{d} \hat{R}\left|\int \rho_{\alpha}(\mathbf{r}) \rho_{\beta}(\hat{R} \mathbf{r}) \mathrm{d} \mathbf{r}\right|^{n}
$$

where the exponent is typically set to $n>1$ to retain angular information. ${ }^{29}$ In practice, it is computationally more efficient to expand the atomic density in a set of orthogonal radial basis functions and spherical harmonics up to a given $n_{\max }$ and $l_{\max }$. The resulting combination coefficients on their own do not yet ensure rotational invariance (because all spherical harmonics with $l>0$ depend on the angular orientation) and are therefore collected into a power spectrum vector. The SOAP kernel may then be calculated by taking the normalised dot product of the two power spectrum vectors associated with each atomic environment, raised to an exponent $\zeta$ which serves to accentuate the distinction between the two environments. ${ }^{28}$

As shown in ref 48 for elemental structures and in ref 39 for a range of inorganic and hybrid materials, geometric similarity may be assessed using SOAP for uniformly re-scaled structures, enabling direct comparison irrespective of characteristic $\mathrm{A}-\mathrm{B}$ distances. We have implemented two scaling approaches: either scaling to a uniform minimum $r(\mathrm{~A}-\mathrm{B})$ distance or scaling to a uniform average $r(\mathbf{A}-\mathbf{B})$ distance.

To extend the similarity measure beyond comparing individual atomic environments, similarities between pairs of atoms in each of the crystal structures are calculated:

$$
\bar{k}\left(\mathcal{A}_{i}, \mathcal{A}_{j}\right)=\frac{1}{N_{\mathcal{A}_{i}} N_{\mathcal{A}_{j}}} \sum_{\alpha \in \mathcal{A}_{i}}^{N_{\mathcal{A}_{i}}} \sum_{\beta \in \mathcal{A}_{j}}^{N_{\mathcal{A}_{j}}} k(\alpha, \beta),
$$

where $\alpha(\beta)$ runs over all atomic sites, $\mathcal{A}$, in the unit cell of structure $i(j)$, respectively. Variations of this method are also implemented where the atomic sites considered are restricted to a given site-type (e.g. A sites), thereby shifting the focus of the similarity analysis towards those particular sites. (In this case, information regarding the other site-types is still implicitly encoded through the neighbor densities.)

\section{Dimensionality Reduction and Visualiza- tion}

To interpret (cg-) SOAP analysis results, the dataset is often visualized as a two-dimensional projection. ${ }^{29,32,39}$ A large number of algorithms are available to carry out this projection (or "embedding"), and a central aspect of the present work will be to compare different widely used embedding schemes. Our implementation stores the similarity of all structures with one another in the form of a symmetric similarity matrix, $\mathbf{K}$, which we construct using the per-cell averaged similarity (Eq. (3)), viz. $K_{i, j}=\bar{k}\left(\mathcal{A}_{i}, \mathcal{A}_{j}\right)$. A corresponding geometric distance matrix, $\mathbf{D}$, may also be defined with elements

$$
D_{i, j}=\sqrt{2-2 K_{i, j}}
$$

to satisfy the triangle inequality. ${ }^{29}$ We currently provide interfaces to the following dimensionality reduction algorithms implemented in external code packages: multi-dimensional scaling (MDS), ${ }^{49}$ t-distributed stochastic neighbor embedding (t-SNE), ${ }^{50}$ and the uniform manifold 
approximation and projection (UMAP). ${ }^{51}$

\section{Bonding and Properties}

During the reduction of MOF structures to their coarse-grained representations, a dictionary of bonds between building units is stored, as recently defined in the IUCr topology dictionary (topoCIF). This enables the CIF output to contain the requisite information to readily construct the underlying net, ${ }^{52,53}$ i.e. the net of building units, as well as to calculate its topological descriptors (e.g., using ToposPro). It also enables the calculation of local-environment properties, including bond lengths, angles, and order parameters. We use a module, referred to as bonding, to extract this geometric information and calculate Chau-Hardwick tetrahedral order parameters and Steinhardt bond order parameters. ${ }^{54-56}$ This module might also be extended for other custom analyses.

Two global structure properties are also included in the routine structure analysis. The first is A-site density, an important material descriptor when considering the potential void space present in a framework. The second property is the A-site SOAP heterogeneity introduced in previous work, ${ }^{39}$ which measures the diversity of the $A$-site environments in a given structure. A value of zero means that all Asite environments are geometrically equivalent (up to the SOAP cut-off radius); a higher value indicates greater diversity. It is calculated as

$$
\mathcal{H}_{\mathrm{A}}=\left(\begin{array}{c}
N_{\mathcal{A}} \\
2
\end{array}\right)^{-1} \sum_{(\alpha, \beta) \in P} k(\alpha, \beta),
$$

where $P$ is the set of (unordered) pairs of distinct A-site environments in the structure, $N_{\mathcal{A}}$ is the number of $\mathbf{A}$-site atoms in the structure, and $k$ is the SOAP similarity kernel defined in Eq. (2).

\section{Database Details}

Primary data were selected from the sample that was prepared for ref 13, filtering for all structures in which the coordination formula was $A B_{2}$ and the ligand (B) was two-connected. The sample contained 1,160 crystal structures, to which 102 structures from the CSD 5.42 update 1 (Feb 2021) were added. A complete list of the 1,262 crystal structures and their topological descriptors is provided in the Supporting Information (see file CF_A_B2_1262.xlsx). The "Experimental literature" to be processed was exported as CIFs from the CSD using the 1,262 entry refcodes and processed unchanged.

\section{Results and Discussion}

\section{Visualizing Geometric Diversity}

We begin by visualizing the geometric diversity in the $\mathrm{AB}_{2} \mathrm{MOF}$ dataset, here embedding cg-SOAP similarities for all (uniformly scaled) structures in two dimensions. Dimensionality reduction is an unsupervised ML task, aiming to extract information from unlabeled datasets, e.g. by clustering the data into groups. We start by visualizing the dataset using one of the simplest algorithms, MDS (as used in ref 39), in Figure 2. The structure map carries the intuitive interpretation that structures that are similar appear close together, and structures that are dissimilar are further apart. In the context of MOFs, structures are generally reported with their topological identifier which uniquely identifies the underlying net. We highlight the distribution of the three most commonly occurring topologies in the dataset - the diamondlike dia net (189 structures), the sodalite-like sod net (136 structures), and the four-fold interpenetrated $\mathbf{4} \#$ dia net (116 structures) - to investigate the degree to which topological variation is represented in the cg-SOAP representation. Strikingly, all three topological families have structures dispersed over the map, with the dia topology being most widely distributed among the three (light blue). There are regions of overlapping points, particularly for dia and sod in the lower part of Figure 2, indicating that topologically dissimilar MOFs might in fact show similarities in terms of their geometric structure.

We quantify the relative distributions of each topology in the map based on how far, on average, the structures of that group are from its respective centroid. These relative distributions are $0.771,0.386$, and 0.441 for dia, sod, 


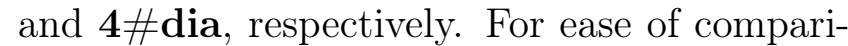
son, we normalize the relative distributions such that the value for dia is unity (Table 1 ).

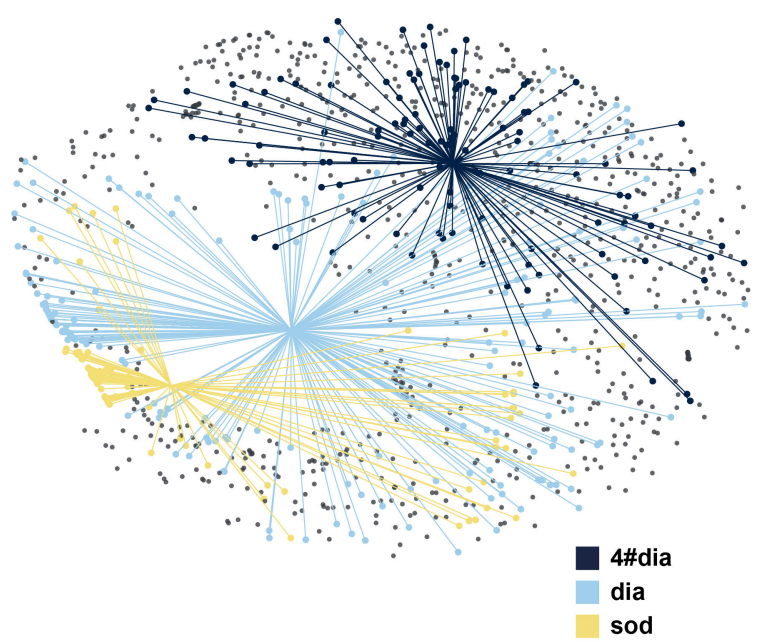

Figure 2 Geometric diversity within isoreticular groups of $A_{2}$ MOFs. The graph shows a twodimensional visualization of the cg-SOAP-based structural distances using an MDS embedding: generally, the closer two points are, the more similar their coarse-grained and re-scaled geometric structures. The distribution of the three most commonly occurring topologies within our dataset (dia, sod, and fourfold-interpenetrated dia, denoted 4\#dia) is emphasized by lines that originate from the respective centroid. Dataset entries with different topologies than the three aforementioned ones are all represented by gray points.

We see that dia networks can be formed with a much larger variety of geometries compared to sod; this is in accordance with the conclusions derived from an analysis of the natural tiles (cages). ${ }^{13}$ This reflects a larger deformability of the diamondoid structures and adaptability to building blocks with geometries spanning a wide range of volumes, lengths, and angles. This feature promotes the dominance of the dia topology in coordination networks. ${ }^{13}$ The folding of the dia networks into interpenetrating arrays, however, significantly restricts the diversity of acceptable network geometries.

Cluster analysis can assist the interpretation of complex datasets by grouping data and identifying representative examples, from which patterns can be more readily identified. Affin- ity propagation is a clustering algorithm that views each data point as a node in a network, and recursively minimizes the edge weights between nodes; the magnitude of each edge at a given time reflects the current affinity the point has for selecting the second point as its "examplar". ${ }^{57}$ Figure 3a shows the dia structures (light blue data points in Figure 2) now divided into four clusters, with the exemplar coarsegrained structures visualized. From these clusters, we investigate the distribution of structural properties in different regions of the map in order to appreciate the geometric diversity available within the dia topology. In the context of MOFs, low metal densities are the simplest indication for the presence of void space: an important feature for catalytic applications. We define the geometric density as the (unitless) density of the structures that have been coarse-grained and scaled (to unity minimum $r(\mathrm{~A}-\mathrm{B})$ bond length). The geometric density is related to the experimental metal density by the characteristic framework bond length. We plot the distribution of the geometric density, average angular component of the ChauHardwick order parameter, $S_{\mathrm{g}}$ (a value of zero corresponds to ideal tetrahedral bond angles; a value of unity would correspond to the extreme case where all four bonds are superimposed), and the average $\mathrm{A}-\mathrm{B}-\mathrm{A}$ angle in Figure $3 \mathrm{~b}-\mathrm{d}$, respectively.

Figure 3b shows the distribution of geometric density for each cluster. There is a relatively clear separation between each group, with cluster $\mathbf{2}$ (orange) containing notably more dense frameworks. It is interesting to note that clusters $\mathbf{2}$ and $\mathbf{4}$, which have higher average geometric density, also seem to have a broader distribution across the MDS map, relative to the narrow distributions of clusters $\mathbf{1}$ and $\mathbf{3}$. This could, in part, be explained by the relatively high deviation away from ideal tetrahedral bond angles about the $A$ sites, as evident from the plot of the angular Chau-Hardwick tetrahedral order parameter, $S_{\mathrm{g}}$ (Figure 3c). Figure 3d shows the distribution of the average $\mathrm{A}-\mathrm{B}-\mathrm{A}$ bond angle; again, clusters $\mathbf{2}$ and $\mathbf{4}$ demonstrate broad distributions with lower average values.

We extend the analysis of the dia subset 
Table 1 Characteristics of the Ten Most Commonly Occurring Topologies in the Dataset

\begin{tabular}{|c|c|c|c|c|c|}
\hline \multirow[b]{2}{*}{ Topology } & \multirow[b]{2}{*}{ Occurrences } & \multirow{2}{*}{$\begin{array}{l}\text { Geometric density }^{a} \\
(\text { mean } \pm \mathrm{SD})\end{array}$} & \multicolumn{3}{|c|}{ Relative distribution } \\
\hline & & & MDS & $\mathrm{t}-\mathrm{SNE}$ & UMAP \\
\hline dia & 189 & $124 \pm 55$ & \multicolumn{3}{|c|}{1 (reference) } \\
\hline sod & 136 & $79 \pm 25$ & 0.50 & 0.45 & 0.59 \\
\hline 4\# dia & 116 & $260 \pm 77$ & 0.57 & 0.47 & 0.40 \\
\hline 5\#dia & 95 & $270 \pm 87$ & 0.67 & 0.48 & 0.50 \\
\hline $3 \#$ dia & 88 & $206 \pm 75$ & 0.71 & 0.48 & 0.42 \\
\hline $2 \#$ dia & 79 & $181 \pm 81$ & 0.91 & 0.66 & 0.50 \\
\hline cds & 41 & $221 \pm 87$ & 0.81 & 0.51 & 0.45 \\
\hline qzd & 38 & $156 \pm 15$ & 0.12 & 0.22 & 0.26 \\
\hline 6\#dia & 36 & $335 \pm 136$ & 0.74 & 0.53 & 0.47 \\
\hline 3\# dmp & 33 & $292 \pm 48$ & 0.38 & 0.23 & 0.31 \\
\hline
\end{tabular}

${ }^{a}$ The geometric density is here defined as $n(\mathbf{A}) \times 1000 / V_{\text {scaled }}$, where $n(\mathbf{A})$ is the number of $\mathrm{A}$ sites in the unit cell, and $V_{\text {scaled }}$ is the volume of the scaled unit cell.

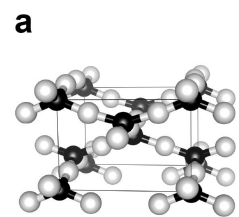

All dia MOFs in dataset

LAJTAR

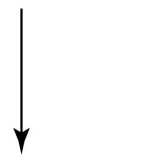

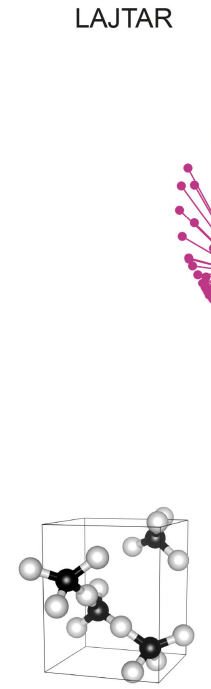

IGEXOH
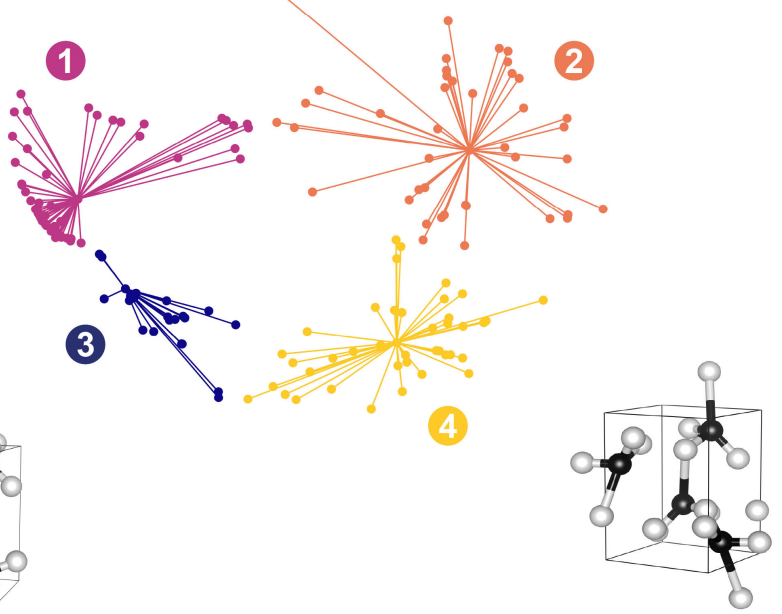

REFGUG

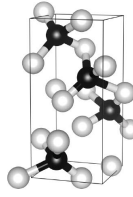

OYEJAE

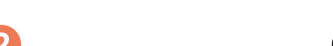

C

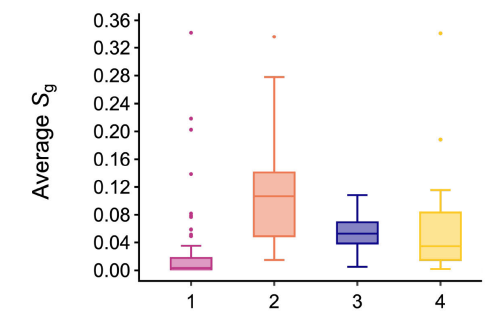

d

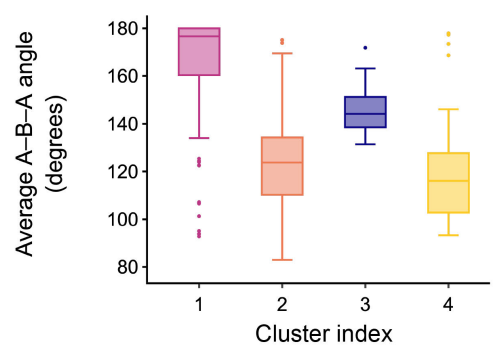

Figure 3 Geometric diversity within MOFs of dia topology. (a) From the dataset characterized in Figure 2, we isolate the dia entries, and analyze their distribution using a clustering algorithm, viz. affinity propagation. ${ }^{57}$ For each cluster, the algorithm selects an "examplar" datapoint, and the corresponding coarse-grained structures and their CSD refcodes are shown. Distributions of local properties for each cluster are presented on the right-hand side: (b) the geometric density; (c) the average angular component of the Chau-Hardwick order parameter, $S_{\mathrm{g}}$, and (d) the average A-B-A angle. Throughout this paper, box plots are drawn such that boxes range from the $25^{\text {th }}$ to the $75^{\text {th }}$ percentile, with the median indicated by a horizontal line; whiskers span \pm 1.5 times the interquartile range, and points outside this range are plotted with circle markers. 
of structures using parameters extracted using ToposPro, ${ }^{53}$ in particular, the tile average distortion $^{13}$ supports the results illustrated in Figure 3. Clusters with higher average geometric density have larger distortions in the tetrahedral coordination of the $A$ sites, corresponding to a collapse of the tiles. Conversely, the most porous structures have A site coordination environments closer to an ideal tetrahedron and the largest proportion of tiles close to the adamantane tile of the ideal dia net. Analogously, structures with $\mathbf{A}-\mathbf{B}-\mathbf{A}$ angles less than $150^{\circ}$ also correspond to denser structures due to the collapse of tiles.

Figure 2 emphasizes that a single topological classification may give rise to a geometrically diverse set of structures; that is to say, one cannot necessarily predict the geometric features of a structure from its topological label alone. From Figure 2, we therefore infer that the study of the latent geometric configurational space may provide insight into a different set of material properties (e.g., bulk modulus) compared to those which are accounted for by the topology (e.g., porosity $\left.^{13}\right)$.

\section{Embedding Schemes}

The universal aim of dimensionality reduction algorithms is to capture meaningful structure in high-dimensional data when embedded into low dimensions. However, it is imperative to consider the algorithm methodology when interpreting the structure map. To illustrate this, we have visualized our dataset with three dimensionality reduction algorithms; namely, MDS, ${ }^{49}$ t-SNE, ${ }^{50}$ and $\mathrm{UMAP}^{51}$ (Figure 4). In order to understand the differences between each representation, we color-code the map by the geometric density (Figure 4a), and by the distribu-

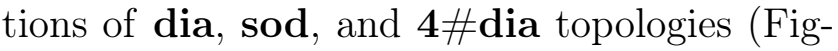
ure 4b, cf. Figure 2), and compare side-by-side how these properties are represented in the results of different embedding schemes.

All three embeddings demonstrate a strong correlation of the distribution of points with geometric density, and qualitatively similar trends in the relative distributions of topologies within each structure map (Table 1). The detail, however, varies. MDS leads to a distinct region in the center of the map where no structures are found. This perhaps suggests that this algorithm, which seeks to minimize a relatively simple loss function, is reaching its limits of usefulness for the size and complexity of the dataset considered here.

The "island-like" features in both the t-SNE and UMAP representations - or more specifically, the absence of islands in the MDS representation - further emphasize this point. The most prominent island (right hand-side of the tSNE map and upper-right of the UMAP map) is a family of qzd MOFs, with the smallest relative distribution, among the ten most commonly occurring topologies in the dataset, for MDS and t-SNE (Table 1). Manual inspection of the structures reveals a high frequency of similar CCDC reference codes (refcodes). The refcode system is designed to group together structures into families, such as the same compound having been crystallized and characterized under different conditions, or polymorphs of the same compound. There are two families of refcodes within the qzd MOF cluster: LIWDEB (13 occurrences) and UKUVOL (21 occurrences). ${ }^{58,59}$ All of these structures are one of two isomers of $\left[\{\mathrm{Cu}\right.$ (succinate $)\left(4,4^{\prime}-\right.$ bipyridine) $\}_{n}$ ], isolated under different experimental conditions, and thus could be considered duplicates. The high geometric similarity between duplicates relative to the similarity between other structures in the dataset skews the representation towards creating an isolated cluster.

\section{Islands and Duplicates}

The occurrence of "islands" of structures, separated out near the edge of the t-SNE and UMAP representations, requires a more subtle interpretation. For example, one of the islands to the left (right) of the t-SNE (UMAP) maps, respectively, corresponds to structures with zni and coi topologies ( $\alpha$ and $\beta$ polymorphs of $\mathrm{Zn}(\mathrm{Im})_{2}$, respectively), predominantly classified within the IMIDZB refcode family. "Duplicates" cannot necessarily be identified as those with a common refcode; however, cg-SOAP screening can help to automate this procedure. To illustrate this point, we isolate the IMIDZB 

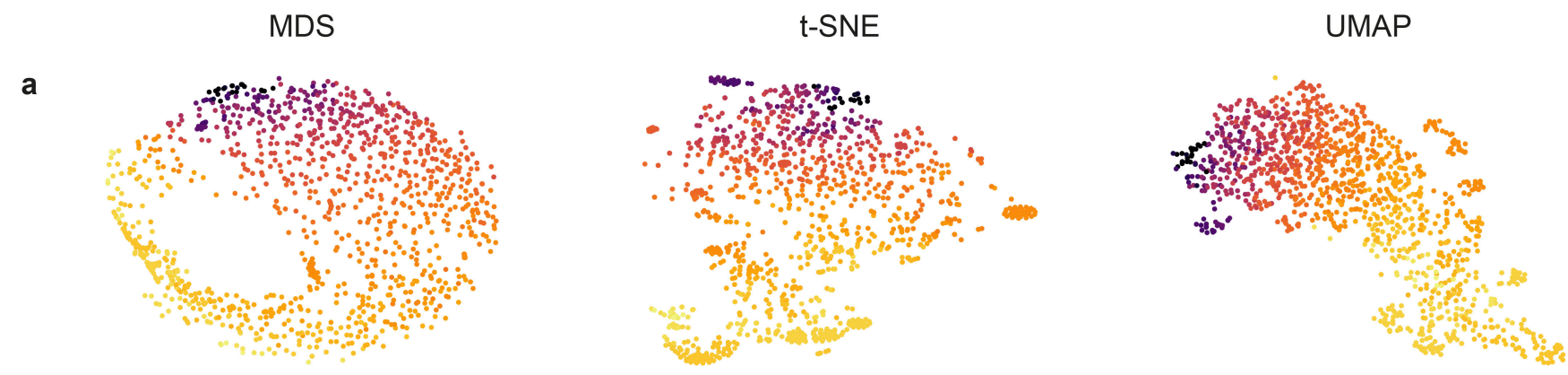

b
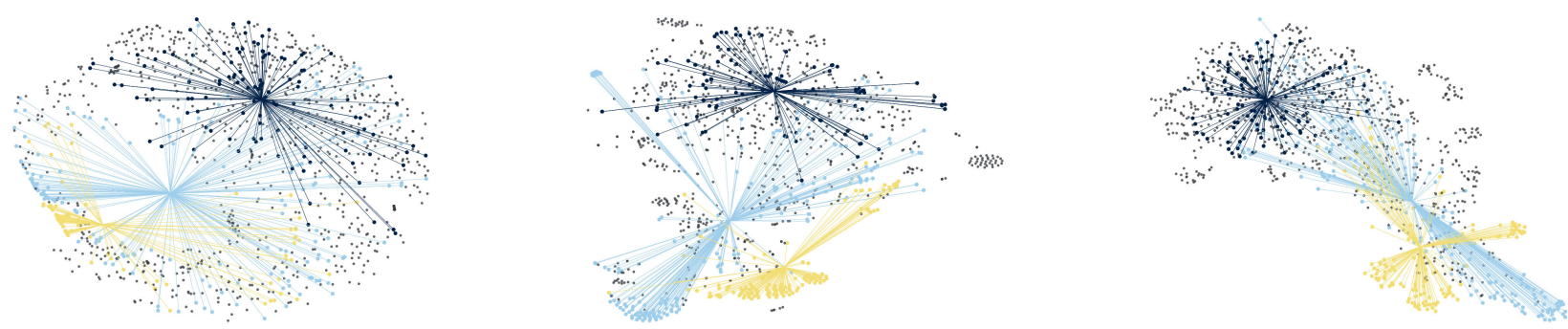

Figure 4 Visualizing geometric diversity in MOFs using different embedding schemes. The plots compare the results of multi-dimensional scaling (MDS; 49 left), $t$-distributed stochastic neighbor embedding (t-SNE; ${ }^{50}$ center), and uniform manifold approximation and projection (UMAP; ${ }^{51}$ right). For each embedding scheme, we show two-dimensional structure maps characterizing the coarse-grained and scaled $\mathrm{AB}_{2} \mathrm{MOF}$ dataset, colored (a) by geometric density, and (b) with the distribution of the three most commonly occurring topologies in the dataset, dia, sod, and $\mathbf{4 \# d i a , ~ h i g h l i g h t i n g ~ t h e ~ r e s p e c t i v e ~ c e n t r o i d s ~ a s ~}$ in Figure 2. 
family of structures from the database, create a structure map using t-SNE (because this algorithm generally achieves clearer clustering of data into distinct regions, albeit at the cost of meaningful inter-cluster distances), and analyze the representation using affinity propagation, as shown in Figure 5.

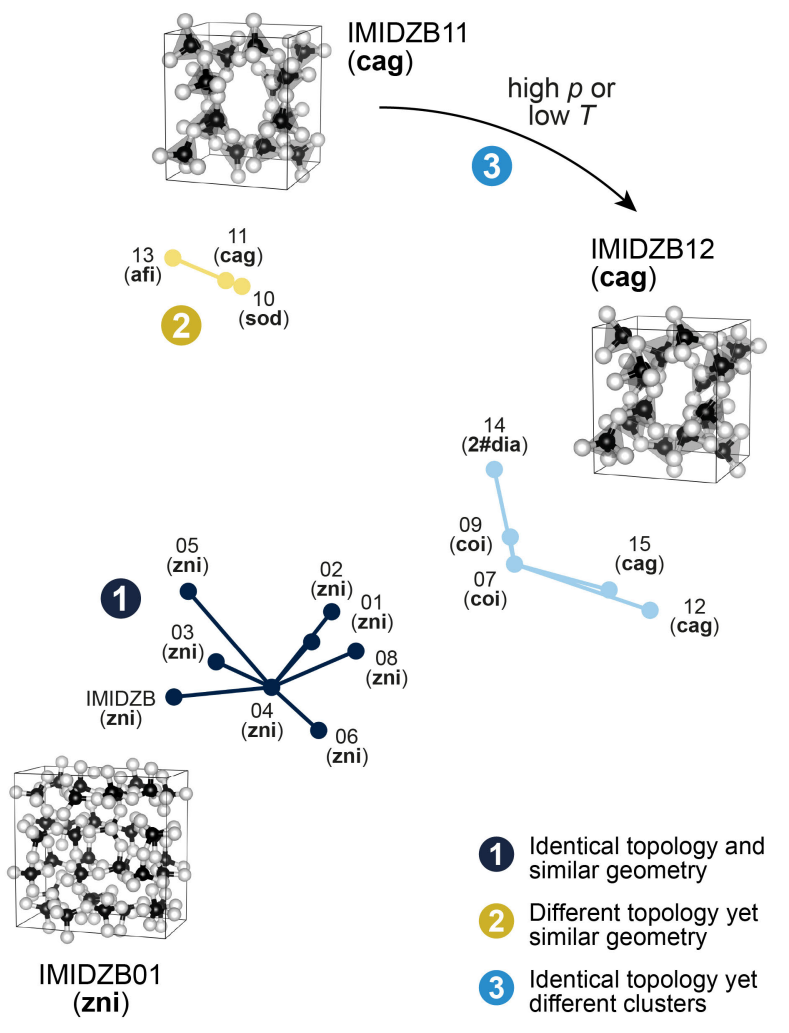

Figure 5 Using cg-SOAP analysis to identify relationships between individual CSD entries with a single refcode. The example case here is given by the polymorphs of $\mathrm{Zn}(\mathrm{Im})_{2}$, with $\mathrm{CSD}$ refcodes IMIDZB, suffixed with numbers representing a running index. The geometric diversity within the IMIDZB refcode family is visualized using t-SNE embedding and a cluster analysis by affinity propagation. The structures are labeled according to topology. The zni polymorphs cluster together; it could therefore be beneficial to choose a representative structure from this cluster. The spread of the cag topology into two distinct clusters illustrates the complexity of "duplicate" detection, as discussed in the text.

The IMIDZB refcode family contains different polymorphs of $\mathrm{Zn}(\mathrm{Im})_{2}$ with different characteristic geometries and topologies, which therefore separate in the cg-SOAP map. By ana- lyzing the geometric diversity in this smaller configuration space, we propose an automated "duplicate" structure identification procedure. The zni topology is the densest, most stable crystalline polymorph of $\mathrm{Zn}(\mathrm{Im})_{2}$, and all structures of this connectivity are found in the same cluster, labeled as 1. One structure (e.g., the cluster examplar selected by the affinity propagation algorithm) could be taken as representative of this particular polymorph. The distribution of cag frameworks across two clusters (2 and $\mathbf{3}$ ), however, is an example where frameworks with identical composition, connectivity, and space-group display diverse geometries. Whereas IMIDZB11 corresponds to the desolvated ZIF-4 framework under ambient conditions (298 K, 1 atm), IMIDZB12 and IMIDZB15 are the same framework after decreasing temperature $(80 \mathrm{~K})$ and increasing pressure $(0.15 \mathrm{GPa})$, respectively, exemplifying the "breathing" effect in the frameworks. ${ }^{60,61}$ With the change of external stimuli, the frameworks become more dense: the average $\mathrm{A}-\mathrm{B}-\mathrm{A}$ angle decreases and $S_{\mathrm{g}}$ increases, corresponding to a lowered "tetrahedrality" around the Zn (A) sites. On these grounds, it may be desirable to keep one structure from each cluster, i.e. IMIDZB10 (cluster 2) and IMIDZB12 or IMIDZB15 (cluster 3), in order to capture the geometric diversity fully.

More generally, one could propose an algorithm that classifies duplicates by considering the refcode, topology, and cg-SOAP similarity as an automated approach that makes it possible to preserve the subtle geometric diversity that arises from varying experimental conditions. The screening of duplicates is expected to be helpful (and indeed required) for moving to very large databases in the future, as demonstrated in a recent analysis of DFT-optimized datasets of MOFs. ${ }^{62}$

\section{Quantifying Geometric Diversity}

Our approach also enables quantitative investigation of local structural properties, and how they are distributed for different categories of structures (e.g., topology). For example, interpenetration is commonly found in MOFs, and it holds implications for the potential function- 
ality of a given compound, because it is closely related with porosity. ${ }^{63}$ Generally, porous materials minimize the energy of the framework through optimal filling of void space, and thus in cases where void space is of sufficient size, interpenetration may be observed. Controlling the degree of interpenetration has been explored using subtle changes to the synthetic methodology, such as varying reaction conditions, templating agents, and ligand design. ${ }^{64,65}$ Given the increasing number of MOF crystal structures reported, the question arises as to whether we can post-rationalize the extent to which the local geometry influences the tendency to interpenetrate.

In Figure 6, we show the distributions of local properties for each structure, for different degrees of interpenetration of the diamond-like net $(Z=1$ corresponds to dia MOFs, $Z=2$ to $\mathbf{2} \# \mathbf{d i a}$, and so on). In Figure 6a, we show the distribution of A-site heterogeneity values (eq $5)$. Figures $6 \mathrm{~b}$ and $6 \mathrm{c}$ illustrate the distributions of established local property descriptors: namely, $S_{\mathrm{g}}$ and the average A-B-A angle, respectively.

We note that the majority of dia MOFs have locally homogeneous A sites, and this homogeneity does not appear to substantially depend on the degree of interpenetration (Figure 6a). Similarly, the distribution of the average $S_{\mathrm{g}}$ does not show a clear correlation with the degree of interpenetration (Figure 6b). There is, by contrast, a much stronger correlation with the $\mathbf{A}-\mathbf{B}-\mathbf{A}$ angle: as the degree of interpenetration increases, the average $A-B-A$ angle tends toward $180^{\circ}$ (Figure 6c). This makes intuitive sense when one considers that longer, "rod-like" ligands gives rise to greater void space and therefore enable a greater degree of interpenetration, and is consistent with the synthetic approach of employing longer spacer ligands to target higher degrees of interpenetration. ${ }^{66-68} \mathrm{It}$ may be inferred from these distributions that the A sites maintain a similar environment, irrespective of the degree of interpenetration, whereas the linker geometry plays a crucial role in determining this property.

Finally, we extend this quantitative analysis to all topologies that occur at least five times in a

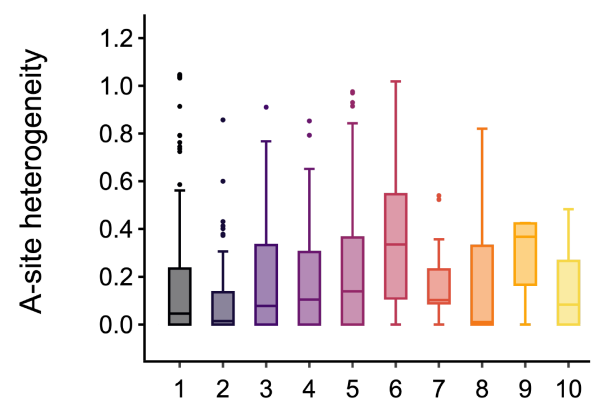

b

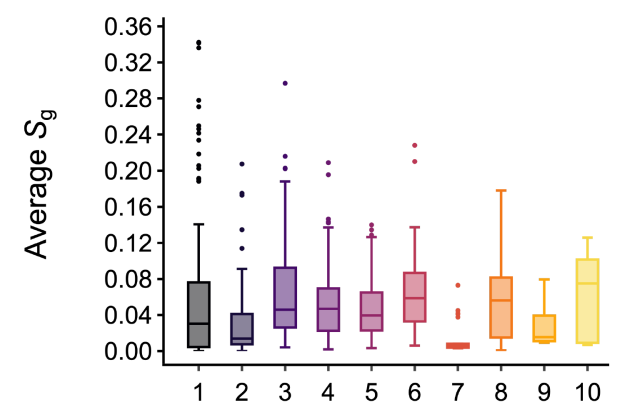

c

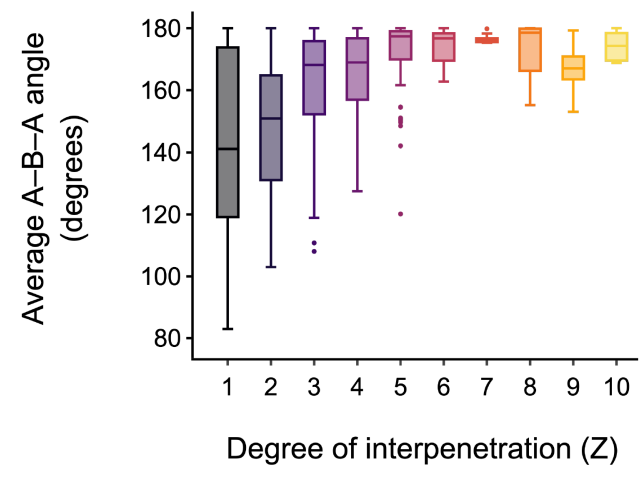

Figure 6 Distributions of geometric property indicators for increasing degrees of interpenetration, $Z$, of the dia-based MOFs in the dataset. The figure shows: (a) the A-site heterogeneity (eq 5), (b) the average angular component, $S_{\mathrm{g}}$, of the ChauHardwick order parameter, and (c) the average A$\mathrm{B}-\mathrm{A}$ angle. 


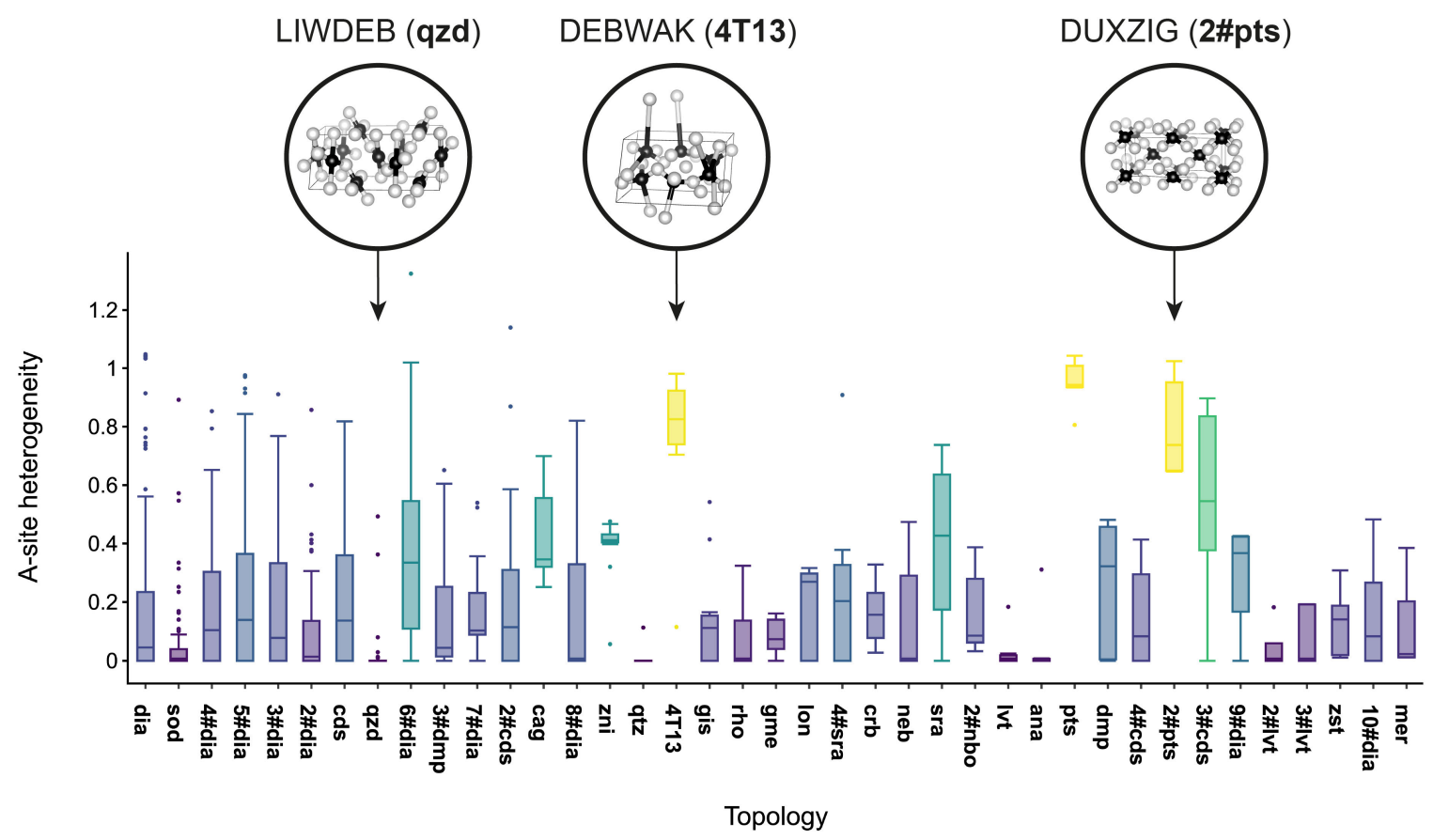

Figure 7 Distributions of A-site heterogeneity (eq 5 ) for all structures in the dataset for which the topology occurs $\geq 5$ times. Each bar is colored according to the average A-site heterogeneity within the given topology. Three example MOFs are highlighted with coarse-grained structural representations, CSD refcodes, and the corresponding topology symbol, and are discussed in the text.

the dataset, plotting the distribution of A-site heterogeneity values in the respective structures in Figure 7.

The qzd structures have a distinctly narrow distribution of $\mathrm{A}$-site heterogeneity values, which reinforces the hypothesis that the separation from the main body of structures in the t-SNE and UMAP embeddings (Figure 4) is a skewing of the visualization as a result of duplicate structures. It is also interesting to note that the zni topology has a narrow A-site heterogeneity distribution at relatively high average value, which likely contributes to the separation of the zni structures.

Figure 7 highlights examples of topologies with particularly low (qzd) and particularly high (4T13, pts, and 2\#pts) A-site heterogeneity, for which illustrative coarse-grained and scaled crystal structures are visualized. The structures with pts and $\mathbf{2} \#$ pts topoology (which are related by increasing from a single to two-fold interpenetrated net) all contain both tetrahedral and square planar geometries about different $\mathbf{A}$ sites, typically by combining
Zn (tetrahedral geometric preference) with any of $\mathrm{Ni}, \mathrm{Cu}, \mathrm{Pt}$, or $\mathrm{Pd}$ (square planar geometric preference). Some frameworks have $\mathrm{Cu}$ in both the tetrahedral and square planar sites of the pts framework. When combined with $\mathrm{Au}, \mathrm{Cu} / \mathrm{Ag}$ occupy the tetrahedral sites in the $\mathbf{2} \#$ pts framework. One might consider attempting to target these pts topologies, therefore, by selecting metals with the appropriate geometric preferences demanded by the framework.

The high degree of heterogeneity in the 4T13 frameworks can be attributed to a large disparity between the lengths of, and the flexibility afforded by using two organic linkers (cf. DEBWAK in Figure 7). The 4T13 frameworks have a short linker (e.g. isophthalate in DEBWAK) and a long-chain, flexible organic linker (e.g. $N, N^{\prime}$-bis(pyridin-4yl)-2,2'-bipyridine-5,5'-dicarboxamide in DEBWAK). ${ }^{69,70}$ The resultant framework has 1-D helical chains that cross each other to create a 2-D molecular braid with geometrically distinct $\mathrm{Zn}$ sites. Automated, quantitative anal- 
ysis such as that exemplified in Figures 6 and 7 should be a helpful part of the methodology used in future work for understanding the geometric and structural diversity in databases of materials.

\section{Conclusions}

We have studied the structures of $A_{2}$ MOFs containing a diverse set of two-connected organic linkers. By coupling a cg-SOAP approach to different embedding schemes, we visualized and analyzed the geometric diversity in a database of MOF structures. With the aid of cluster analysis, the structure maps of the $\mathrm{AB}_{2}$ MOF configuration space can be better understood. Here, we focused on clustering within the low-dimensional embedding, and demonstrated how the location of structures in the map was consistent with the grouping of structures with similar structural properties. We described the cg-SOAP and visualization methodology implemented in a Python package, and validated the routines by confirming the underlying net was correct, using the ToposPro software. We anticipate that the methodology described in this work will be useful in visualizing, analyzing, and understanding the geometric diversity in larger MOF datasets, to which we will dedicate future work.

\section{Computational Details}

Our code imports functionality from Pymatgen, ${ }^{41}$ the Atomic Simulation Environment (ASE), ${ }^{71}$ and Network $X^{47}$ for structure processing tasks. The SOAP implementation is imported from DScribe. ${ }^{72}$ Scikit-learn, ${ }^{73}$ t-SNE, ${ }^{50}$ and $\mathrm{UMAP}^{51}$ are used for dimensionality reduction. The Scikit-learn package implementation of affinity propagation was used for cluster analysis.

All structures were uniformly scaled to a minimum $r(\mathrm{~A}-\mathrm{B})$ bond distance of unity. We computed SOAP vectors using the polynomial basis functions implemented in DScribe, with a radial cut-off of $r_{\text {cut }}=2.5$, smoothness of $\sigma=0.2$, and atomic neighbor density expansion up to $n_{\max }=10, l_{\max }=9($ as in ref 39$)$. (Note that here, we do not include units for $r_{\text {cut }}$ and $\sigma$ be- cause we have re-scaled all structures.)

In terms of technical comparisons of the different algorithms, it is worth mentioning the relative times taken for the code to execute. All three algorithms could be performed on a standard MacBook Pro (1.4 GHz Quad-Core Intel Core i5 processor; 8 GB memory). The absolute times for MDS, t-SNE, and UMAP were 103, 479, and 22 seconds, respectively. Hence, UMAP outperforms the other two embedding schemes for this particular purpose. It should be noted that a particularly low learning rate, 5 , was chosen for t-SNE, because this was found to better capture the structure of the data (based on visual inspection of the relative "tightness" of clustering: smeared-out clusters can often be a sign that the algorithm has ended before reaching convergence). For future work on larger datasets, UMAP might therefore be preferred over t-SNE for its faster execution time.

The absolute positions of data points in the structure maps will slightly depend on the specific parameters chosen (and numerical issues), particularly for t-SNE and UMAP; however, the global trends and interpretation of the visualizations were found to remain consistent for different choices of embedding parameters.

\section{Data and Code Availability}

The coarse-graining code described in this work is openly available online at https://github.com/tcnicholas/coarse-graining. The full dataset of coarse-grained structures will be made available via Zenodo upon journal publication.

Acknowledgement T.C.N. was supported by the EPSRC (DTP award EP/T517811/1) and the European Research Council (ERC Advanced Grant 788144, to A.L.G.). E.V.A. is grateful to the Russian Science Foundation for supporting topological methods of crystal structures analysis with the program package ToposPro (Grant No. 18-73-10116). V.A.B. thanks the Russian Foundation for Basic Research for support (Grant No. 18-29-04010). 


\section{References}

(1) Groom, C. R.; Bruno, I. J.; Lightfoot, M. P.; Ward, S. C. The Cambridge Structural Database. Acta. Cryst. B 2016, 72, 171-179.

(2) Zagorac, D.; Müller, H.; Ruehl, S.; Zagorac, J.; Rehme, S. Recent developments in the Inorganic Crystal Structure Database: theoretical crystal structure data and related features. J. Appl. Cryst. 2019, 52, 918-925.

(3) Butler, K. T.; Davies, D. W.; Cartwright, H.; Isayev, O.; Walsh, A. Machine learning for molecular and materials science. Nature 2018, 559, 547-555.

(4) Jablonka, K. M.; Ongari, D.; Moosavi, S. M.; Smit, B. Big-Data Science in Porous Materials: Materials Genomics and Machine Learning. Chem. Rev. 2020, 120, 8066-8129.

(5) Rosen, A. S.; Iyer, S. M.; Ray, D.; Yao, Z.; Aspuru-Guzik, A.; Gagliardi, L.; Notestein, J. M.; Snurr, R. Q. Machine learning the quantum-chemical properties of metal-organic frameworks for accelerated materials discovery. Matter 2021, 4, $1578-1597$.

(6) Eddaoudi, M.; Moler, D. B.; Li, H.; Chen, B.; Reineke, T. M.; O’Keeffe, M.; Yaghi, O. M. Modular Chemistry: Secondary Building Units as a Basis for the Design of Highly Porous and Robust Metal-Organic Carboxylate Frameworks. Acc. Chem. Res. 2001, 34, 319-330.

(7) Yaghi, O. M.; O’Keeffe, M.; Ockwig, N. W.; Chae, H. K.; Eddaoudi, M.; Kim, J. Reticular synthesis and the design of new materials. Nature 2003, 423, 705714 .

(8) Long, J. R.; Yaghi, O. M. The pervasive chemistry of metal-organic frameworks. Chem. Soc. Rev. 2009, 38, 1213-1214.
(9) Tranchemontagne, D. J.; MendozaCortés, J. L.; O'Keeffe, M.; Yaghi, O. M. Secondary building units, nets and bonding in the chemistry of metal-organic frameworks. Chem. Soc. Rev. 2009, 38, 1257-1283.

(10) Blatov, V. A.; Shevchenko, A. P.; Proserpio, D. M. Applied Topological Analysis of Crystal Structures with the Program Package ToposPro. Cryst. Growth Des. 2014, 14, 3576-3586.

(11) Delgado-Friedrichs, O.; O'Keeffe, M. Identification of and symmetry computation for crystal nets. Acta Crystallogr., Sect. A 2003, 59, 351-360.

(12) Amombo Noa, F. M.; Svensson Grape, E.; Brülls, S. M.; Cheung, O.; Malmberg, P.; Inge, A. K.; McKenzie, C. J.; Mårtensson, J.; Öhrström, L. Metal-Organic Frameworks with Hexakis(4-carboxyphenyl)benzene: Extensions to Reticular Chemistry and Introducing Foldable Nets. J. Am. Chem. Soc. 2020, 142, 9471-9481.

(13) Shevchenko, A. P.; Alexandrov, E. V.; Golov, A. A.; Blatova, O. A.; Duyunova, A. S.; Blatov, V. A. Topology versus porosity: what can reticular chemistry tell us about free space in metal-organic frameworks? Chem. Commun. 2020, 56, 9616-9619.

(14) Ortiz, A. U.; Boutin, A.; Fuchs, A. H.; Coudert, F.-X. Anisotropic Elastic Properties of Flexible Metal-Organic Frameworks: How Soft are Soft Porous Crystals? Phys. Rev. Lett. 2012, 109, 195502.

(15) Ortiz, A. U.; Boutin, A.; Fuchs, A. H.; Coudert, F.-X. Metal-organic frameworks with wine-rack motif: What determines their flexibility and elastic properties? $J$. Chem. Phys. 2013, 138, 174703.

(16) Eddaoudi, M.; Kim, J.; Rosi, N.; Vodak, D.; Wachter, J.; O'Keeffe, M.; Yaghi, O. M. Systematic Design of Pore 
Size and Functionality in Isoreticular MOFs and Their Application in Methane Storage. Science 2002, 295, 469-472.

(17) Alezi, D.; Belmabkhout, Y.; Suyetin, M.; Bhatt, P. M.; Weseliński, L. u. J.; Solovyeva, V.; Adil, K.; Spanopoulos, I.; Trikalitis, P. N.; Emwas, A.-H.; Eddaoudi, M. MOF Crystal Chemistry Paving the Way to Gas Storage Needs: Aluminum-Based soc-MOF for $\mathrm{CH}_{4}, \mathrm{O}_{2}$, and $\mathrm{CO}_{2}$ Storage. J. Am. Chem. Soc. 2015, 137, 13308-13318.

(18) Chen, Z.; Weseliński, L. J.; Adil, K.; Belmabkhout, Y.; Shkurenko, A.; Jiang, H.; Bhatt, P. M.; Guillerm, V.; Dauzon, E.; Xue, D.-X.; O'Keeffe, M.; Eddaoudi, M. Applying the Power of Reticular Chemistry to Finding the Missing alb-MOF Platform Based on the (6,12)-Coordinated Edge-Transitive Net. J. Am. Chem. Soc. 2017, 139, 3265-3274.

(19) Collings, I. E.; Tucker, M. G.; Keen, D. A.; Goodwin, A. L. Geometric switching of linear to area negative thermal expansion in uniaxial metal-organic frameworks. CrystEngComm 2014, 16, 34983506 .

(20) Sadeghi, A.; Ghasemi, S. A.; Schaefer, B.; Mohr, S.; Lill, M. A.; Goedecker, S. Metrics for measuring distances in configuration spaces. J. Chem. Phys. 2013, 139, 184118.

(21) Zhu, L.; Amsler, M.; Fuhrer, T.; Schaefer, B.; Faraji, S.; Rostami, S.; Ghasemi, S. A.; Sadeghi, A.; Grauzinyte, M.; Wolverton, C.; Goedecker, S. A fingerprint based metric for measuring similarities of crystalline structures. J. Chem. Phys. 2016, 144, 034203.

(22) Musil, F.; Grisafi, A.; Bartók, A. P.; Ortner, C.; Csányi, G.; Ceriotti, M. Physicsinspired structural representations for molecules and materials. arXiv 2021, 2101.04673 .
(23) Behler, J. Atom-centered symmetry functions for constructing high-dimensional neural network potentials. J. Chem. Phys. 2011, 134, 074106.

(24) Rupp, M.; Tkatchenko, A.; Müller, K.-R.; von Lilienfeld, O. A. Fast and Accurate Modeling of Molecular Atomization Energies with Machine Learning. Phys. Rev. Lett. 2012, 108, 058301.

(25) Isayev, O.; Oses, C.; Toher, C.; Gossett, E.; Curtarolo, S.; Tropsha, A. Universal fragment descriptors for predicting properties of inorganic crystals. Nat. Commun. 2017, 8, 15679.

(26) Willatt, M. J.; Musil, F.; Ceriotti, M. Feature optimization for atomistic machine learning yields a data-driven construction of the periodic table of the elements. Phys. Chem. Chem. Phys. 2018, 20, 2966129668.

(27) Willatt, M. J.; Musil, F.; Ceriotti, M. Atom-density representations for machine learning. J. Chem. Phys. 2019, 150, 154110 .

(28) Bartók, A. P.; Kondor, R.; Csányi, G. On representing chemical environments. Phys. Rev. B 2013, 87, 184115.

(29) De, S.; Bartók, A. P.; Csányi, G.; Ceriotti, M. Comparing molecules and solids across structural and alchemical space. Phys. Chem. Chem. Phys. 2016, 18, 13754-13769.

(30) Moosavi, S. M.; Jablonka, K. M.; Smit, B. The Role of Machine Learning in the Understanding and Design of Materials. $J$. Am. Chem. Soc. 2020, 142, 20273-20287.

(31) Moosavi, S. M.; Nandy, A.; Jablonka, K. M.; Ongari, D.; Janet, J. P.; Boyd, P. G.; Lee, Y.; Smit, B.; Kulik, H. J. Understanding the diversity of the metal-organic framework ecosystem. Nat. Commun. 2020, 11, 4068. 
(32) Cheng, B.; Griffiths, R.-R.; Wengert, S.; Kunkel, C.; Stenczel, T.; Zhu, B.; Deringer, V. L.; Bernstein, N.; Margraf, J. T.; Reuter, K.; Csanyi, G. Mapping Materials and Molecules. Acc. Chem. Res. 2020, 53, 1981-1991.

(33) Pulido, A. et al. Functional materials discovery using energy-structure-function maps. Nature 2017, 543, 657-664.

(34) Engel, E. A.; Anelli, A.; Ceriotti, M.; Pickard, C. J.; Needs, R. J. Mapping uncharted territory in ice from zeolite networks to ice structures. Nat. Commun. 2018, 9, 2173.

(35) Yang, J.; De, S.; Campbell, J. E.; Li, S.; Ceriotti, M.; Day, G. M. Large-Scale Computational Screening of Molecular Organic Semiconductors Using Crystal Structure Prediction. Chem. Mater. 2018, 30, 43614371.

(36) Musil, F.; De, S.; Yang, J.; Campbell, J. E.; Day, G. M.; Ceriotti, M. Machine learning for the structure-energy-property landscapes of molecular crystals. Chem. Sci. 2018, 9, 1289-1300.

(37) Helfrecht, B. A.; Semino, R.; Pireddu, G.; Auerbach, S. M.; Ceriotti, M. A new kind of atlas of zeolite building blocks. J. Chem. Phys. 2019, 151, 154112.

(38) Zhao, C.; Chen, L.; Che, Y.; Pang, Z.; Wu, X.; Lu, Y.; Liu, H.; Day, G. M.; Cooper, A. I. Digital navigation of energy-structure-function maps for hydrogen-bonded porous molecular crystals. Nat. Commun. 2021, 12, 817.

(39) Nicholas, T. C.; Goodwin, A. L.; Deringer, V. L. Understanding the geometric diversity of inorganic and hybrid frameworks through structural coarse-graining. Chem. Sci. 2020, 11, 12580-12587.

(40) Jablonka, K. M.; Ongari, D.; Moosavi, S. M.; Smit, B. Using collective knowledge to assign oxidation states of metal cations in metal-organic frameworks. Nat. Chem. 2021, in press, DOI: $10.1038 / \mathrm{s} 41557-021-00717-\mathrm{y}$.

(41) Ong, S. P.; Richards, W. D.; Jain, A.; Hautier, G.; Kocher, M.; Cholia, S.; Gunter, D.; Chevrier, V. L.; Persson, K. A.; Ceder, G. Python Materials Genomics (pymatgen): A robust, opensource python library for materials analysis. Comp. Mater. Sci. 2013, 68, 314-319.

(42) Heller, S. R.; McNaught, A.; Pletnev, I.; Stein, S.; Tchekhovskoi, D. InChI, the IUPAC International Chemical Identifier. J. Cheminf. 2015, 7, 23.

(43) Zhang, J.; Wu, T.; Zhou, C.; Chen, S.; Feng, P.; Bu, X. Zeolitic Boron Imidazolate Frameworks. Angew. Chem., Int. Ed. 2009, 48, 2542-2545.

(44) Yaghi, O. M.; Li, G.; Li, H. Selective binding and removal of guests in a microporous metal-organic framework. Nature 1995, 378, 703-706.

(45) Zimmermann, N. E. R.; Jain, A. Local structure order parameters and site fingerprints for quantification of coordination environment and crystal structure similarity. $R S C A d v$. 2020, 10, 6063-6081.

(46) Joshi, S. Y.; Deshmukh, S. A. A review of advancements in coarse-grained molecular dynamics simulations. Mol. Simul. 2020, $1-18$.

(47) Hagberg, A. A.; Schult, D. A.; Swart, P. J. Exploring Network Structure, Dynamics, and Function using NetworkX. Proceedings of the 7th Python in Science Conference. Pasadena, CA USA, 2008; pp 11-15.

(48) Bernstein, N.; Csányi, G.; Deringer, V. L. De novo exploration and self-guided learning of potential-energy surfaces. npj Comput. Mater. 2019, 5, 99.

(49) Torgerson, W. S. Multidimensional scaling: I. Theory and method. Psychometrika 1952, 17, 401-419. 
(50) Maaten, L. v. d.; Hinton, G. Visualizing Data using t-SNE. J. Mach. Learn. Res. 2008, 9, 2579-2605.

(51) McInnes, L.; Healy, J.; Saul, N.; Großberger, L. UMAP: Uniform Manifold Approximation and Projection. J. Open Source Softw. 2018, 3, 861.

(52) Alexandrov, E. V.; Blatov, V. A.; Kochetkov, A. V.; Proserpio, D. M. Underlying nets in three-periodic coordination polymers: topology, taxonomy and prediction from a computer-aided analysis of the Cambridge Structural Database. CrystEngComm 2011, 13, 3947-3958.

(53) Alexandrov, E. V.; Shevchenko, A. P.; Blatov, V. A. Topological Databases: Why Do We Need Them for Design of Coordination Polymers? Cryst. Growth Des. 2019, 19, 2604-2614.

(54) Chau, P.; Hardwick, A. J. A new order parameter for tetrahedral configurations. Mol. Phys. 1998, 93, 511-518.

(55) Steinhardt, P. J.; Nelson, D. R.; Ronchetti, M. Bond-orientational order in liquids and glasses. Phys. Rev. B 1983, 28, 784-805.

(56) Lechner, W.; Dellago, C. Accurate determination of crystal structures based on averaged local bond order parameters. $J$. Chem. Phys. 2008, 129, 114707.

(57) Frey, B. J.; Dueck, D. Clustering by Passing Messages Between Data Points. Science 2007, 315, 972-976.

(58) Ying, S.-M.; Mao, J.-G.; Sun, Y.Q.; Zeng, H.-Y.; Dong, Z.-C. Syntheses and crystal structures of three openframeworks of metal succinates containing a 4,4'-bipyridine ligand. Polyhedron 2003, 22, 3097-3103.

(59) Wu, S.-T.; Wu, Y.-R.; Kang, Q.-Q.; Zhang, H.; Long, L.-S.; Zheng, Z.; Huang, R.-B.; Zheng, L.-S. Chiral Symmetry Breaking by Chemically Manipulating
Statistical Fluctuation in Crystallization. Angew. Chem., Int. Ed. 2007, 46, 84758479 .

(60) Wharmby, M. T.; Henke, S.; Bennett, T. D.; Bajpe, S. R.; Schwedler, I.; Thompson, S. P.; Gozzo, F.; Simoncic, P.; Mellot-Draznieks, C.; Tao, H.; Yue, Y.; Cheetham, A. K. Extreme Flexibility in a Zeolitic Imidazolate Framework: Porous to Dense Phase Transition in Desolvated ZIF-4. Angew. Chem., Int. Ed. 2015, 54, 6447-6451.

(61) Widmer, R. N.; Lampronti, G. I.; Chibani, S.; Wilson, C. W.; Anzellini, S.; Farsang, S.; Kleppe, A. K.; Casati, N. P. M.; MacLeod, S. G.; Redfern, S. A. T.; Coudert, F.-X.; Bennett, T. D. Rich Polymorphism of a Metal-Organic Framework in Pressure-Temperature Space. J. Am. Chem. Soc. 2019, 141, 9330-9337.

(62) Barthel, S.; Alexandrov, E. V.; Proserpio, D. M.; Smit, B. Distinguishing Metal-Organic Frameworks. Cryst. Growth Des. 2018, 18, 1738-1747.

(63) Jiang, H.-L.; Makal, T. A.; Zhou, H.-C. Interpenetration control in metal-organic frameworks for functional applications. Coord. Chem. Rev. 2013, 257, 2232-2249.

(64) Verma, G.; Butikofer, S.; Kumar, S.; Ma, S. Regulation of the Degree of Interpenetration in Metal-Organic Frameworks. Top. Curr. Chem. 2019, 378, 4.

(65) Gupta, M.; Vittal, J. J. Control of interpenetration and structural transformations in the interpenetrated MOFs. Coord. Chem. Rev. 2021, 435, 213789.

(66) Carlucci, L.; Ciani, G.; Proserpio, D. M.; Rizzato, S. Three Novel Interpenetrating Diamondoid Networks from Self-Assembly of 1,12-Dodecanedinitrile with Silver(I) Salts. Chem. - Eur. J. 2002, 8, 15191526 .

(67) Tian, A.-X.; Ying, J.; Peng, J.; Sha, J.Q.; Pang, H.-J.; Zhang, P.-P.; Chen, Y.; 
Zhu, M.; Su, Z.-M. Tuning the Dimensionality of the Coordination Polymer Based on Polyoxometalate by Changing the Spacer Length of Ligands. Cryst. Growth Des. 2008, 8, 3717-3724.

(68) He, Y.-P.; Tan, Y.-X.; Zhang, J. Deliberate design of a neutral heterometallic organic framework containing a record 25-fold interpenetrating diamondoid network. CrystEngComm 2012, 14, 63596361 .

(69) Jacobs, T.; Clowes, R.; Cooper, A. I.; Hardie, M. J. A Chiral, Self-Catenating and Porous Metal-Organic Framework and its Post-Synthetic Metal Uptake. Angew. Chem. Int. Ed. 2012, 51, 51925195.

(70) Luo, F.; Wang, M.-S.; Luo, M.-B.; Sun, G.-M.; Song, Y.-M.; Li, P.-X.; Guo, G.-C. Functionalizing the pore wall of chiral porous metal-organic frameworks by distinct $-\mathrm{H},-\mathrm{OH},-\mathrm{NH}_{2},-\mathrm{NO}_{2}$, $-\mathrm{COOH}$ shutters showing selective adsorption of $\mathrm{CO}_{2}$, tunable photoluminescence, and direct white-light emission. Chem. Commun. 2012, 48, 5989-5991.

(71) Larsen, A. H. et al. The atomic simulation environment - a Python library for working with atoms. J. Phys.: Condens. Matter 2017, 29, 273002.

(72) Himanen, L.; Jäger, M. O. J.; Morooka, E. V.; Federici Canova, F.; Ranawat, Y. S.; Gao, D. Z.; Rinke, P.; Foster, A. S. DScribe: Library of descriptors for machine learning in materials science. Comput. Phys. Commun. 2020, 247 , 106949 .

(73) Pedregosa, F. et al. Scikit-learn: Machine Learning in Python. J. Mach. Learn. Res. 2011, 12, 2825-2830. 